\title{
Multi-functional microparticles with stimulation and sensing capabilities for facile NK cell activity assay
}

\author{
Jeehun Park ${ }^{\dagger, \#}$, Yerin Shin ${ }^{\ddagger}, \#$, Jung Min Kim ${ }^{\S}$, SoonHo Kweon ${ }^{\ddagger}$, Ah Young Song ${ }^{\S}$, Yujin \\ Baek", Jinho Kim», Duck Cho ${ }^{\dagger+, ~ *}$, Hun Sik Kim ${ }^{\S, *}$ and Junsang Doh ${ }^{\dagger, ~ " I, ~ * ~}$ \\ ${ }^{\dagger}$ Research Institute of Advanced Materials, Seoul National University, Seoul, South Korea \\ ${ }^{\star}$ School of Interdisciplinary Bioscience and Bioengineering (I-Bio), Pohang University of \\ Science and Technology, Pohang, Gyeongbuk, South Korea \\ ${ }^{\S}$ Department of Biomedical Sciences, Asan Medical Center, University of Ulsan College of \\ Medicine, Seoul, South Korea \\ "Department of Materials Science and Engineering, Seoul National University, Seoul, South \\ Korea \\ IDepartment of Health Sciences and Technology, SAIHST, Sungkyunkwan University, \\ Seoul, South Korea \\ ${ }^{+\dagger}$ Department of Laboratory Medicine and Genetics, Samsung Medical Center, \\ Sungkyunkwan University School of Medicine, Seoul, South Korea
}

\section{Supporting Information (SI)}

\section{MATERIALS AND METHODS}

NK cell. All experiments related with human primary cells were approved by Institutional Review Board in Samsung Medical Center (No. SMC 2018-02-102). Primary human NK cells were expanded from peripheral blood mononuclear cells (PBMCs) by co-culture with irradiated K562 cells.[1] Expanded NK cells were cultured in RPMI-1640 supplemented with $10 \% \mathrm{FBS}, 1 \%$ penicillin/streptomycin, $2 \mathrm{mM}$ L-glutamine, $100 \mathrm{U} / \mathrm{ml}$ recombinant human IL-2 and $5 \mathrm{ng} / \mathrm{ml}$ recombinant human IL-15. These cells were $80 \sim 99 \% \mathrm{CD}^{-} \mathrm{CD}^{-} 6^{+}$, as assessed by flow cytometry.

Granzyme B sensor. Granzyme B peptide sensor was synthesized by Peptron, inc. A granzyme $\mathrm{B}$ cleavable peptide with the amino acid sequence VGPDFGR was synthesized, and $\mathrm{N}$ - and Cterminal residues of the peptide were modified with NHS-PEG4-Biotin (Thermo Fisher scientific) and Alexa Fluor 488 NHS Ester (Invitrogen), respectively. 
Fabrication of Multi-functional microparticles for NK cell activity Assays (MNAs). MNAs were fabricated by modifying surfaces of Dynabeads M-450 epoxy $\left(1 \mathrm{ml} ; 4.0 \times 10^{8}\right.$ beads $/ \mathrm{ml}$; Thermo Fisher Scientific). Dynabeads was washed and resuspended in $1 \mathrm{ml}$ buffer containing $0.1 \mathrm{M}$ sodium phosphate and $1 \mathrm{M}$ ammonium sulfate with $\mathrm{pH}$ 7.4. Streptavidin $(40 \mu \mathrm{l} ; 5 \mathrm{mg} / \mathrm{ml}$; Invitrogen) was added to the Dynabead suspension, and incubated for $24 \mathrm{~h}$ at room temperature (RT) with gentle rotation for conjugation. Unreacted epoxy groups on the Dynabeads were quenched by adding $1 \mathrm{ml}$ of Tris buffer ( $100 \mathrm{mM}$ of Tris; $\mathrm{pH} \mathrm{7.9)}$ to the reaction mixture, and incubating for $1 \mathrm{~h}$ at RT. Streptavidin-coated Dynabeads were wash 3 times in PBS, resuspended in bicarbonate buffer ( $100 \mathrm{mM}$ sodium bicarbonate; $\mathrm{pH} 8.3)$ and further labeled with Alexa Fluor 647 by adding $0.2 \mu \mathrm{l}$ of Alexa Fluor 647 NHS Ester $(1 \mathrm{mg} / \mathrm{ml}$ in DMSO; Invitrogen). After $1 \mathrm{~h}$ of incubation at RT, unreacted dye was removed by washing the labeled Dynabeads with PBS. MNAs were constructed by mixing $1 \mathrm{ml}$ of the streptavidin-coated and Alexa 647-labeled Dynabeads with biotinylated anti-NKG2D $(10 \mu \mathrm{l} ; 0.5 \mathrm{mg} / \mathrm{ml}$; R\&D system; clone: 149810), biotinylated anti-2B4 $(10 \mu \mathrm{l} ; 0.5 \mathrm{mg} / \mathrm{ml}$; Invitrogen, clone: eBioC1.7), granzyme B peptide sensor $(10 \mu \mathrm{l} ; 0.5 \mathrm{mg} / \mathrm{ml})$, and biotinylated anti-IFN- $\gamma(10 \mu \mathrm{l} ; 1 \mathrm{mg} / \mathrm{ml}$; Abcam, clone: MD-1) for 10 min at RT, and washing with PBS.

Flow cytometry analysis of MNAs. Fluorescence intensities of MNAs treated with various concentrations of natural human granzyme B protein (Abcam) or recombinant human interferon- $\gamma$ protein (Gibco) in the presence of PE anti-IFN- $\gamma(2 \mu \mathrm{g} / \mathrm{ml}$; BioLegend, clone: 4S.B3) were measured using LSR Fortessa analyzer (BD Biosciences), and data were analyzed with FlowJo. To assess NK cell functions, NK cells treated with Human BD Fc Block antibody $\left(0.5 \mathrm{mg} / \mathrm{ml}\right.$; BD Pharmingen) were used. NK cells $\left(4.0 \times 10^{6}\right)$ were mixed with the same number of MNAs in $50 \mu \mathrm{l}$ of IMDM (Cellgro) supplemented with $10 \%$ FBS with $2 \mu \mathrm{g} / \mathrm{ml} \mathrm{PE}$ anti-human IFN- $\gamma$ antibody for $4 \mathrm{~h}$ at $37^{\circ} \mathrm{C}$ with rotation. After washing NK cell/MNA mixture for 3 times with PBS, flow cytometry was performed to analyze fluorescence intensities of MNAs. Fluorescence intensity changes of granzyme B sensor and IFN- $\gamma$ detection were calculated using formulas described in Figure S5 and S6, respectively.

Standard cytotoxicity assay. NK cell cytotoxicity was measured by a flow cytometry-based assay using Carboxyfluorescein Diacetate Succinimidyl Ester (CFSE) (Invitrogen) staining of K562 target cells. Briefly, K562 cells were stained with $0.5 \mu \mathrm{M}$ CFSE in PBS buffer for 10 min at $37^{\circ} \mathrm{C}$. and washed using complete media. CFSE-stained K562 cells $\left(1 \times 10^{5}\right)$ were placed in a 96-well U-bottom plate and mixed with NK cells (1:1 effector-to-target (E:T) ratios). The plates were centrifuged at $150 \mathrm{~g}$ for $3 \mathrm{~min}$ and incubated for $4 \mathrm{~h}$ at $37^{\circ} \mathrm{C}$ in a $5 \% \mathrm{CO}_{2}$ incubator. The mixed cells were transferred to FACS tubes. After adding $1 \mu \mathrm{l}$ of propidium iodide (PI, 1 $\mathrm{mg} / \mathrm{ml}$, SigmaAldrich) to each tube to stain dead cells, flow cytometry was performed.

ELISA. To prepare the ELISA test, microparticles were constructed with the same composition as MNAs with the replacement of granzyme B sensor and biotinylated anti-IFN- $\gamma$ antibody to biotinylated mouse IgG. Then Fc blocked NK cells $\left(4.0 \times 10^{6}\right)$ were incubated with the same number of microparticles in $50 \mu \mathrm{l}$ of IMDM (Cellgro) supplemented with $10 \%$ FBS for $4 \mathrm{~h}$ at $37^{\circ} \mathrm{C}$ with rotation, and supernatants were collected and analyzed with IFN- $\gamma$ ELISA kit (Thermo Fisher Scientific)

Live cell imaging. A modified Olympus IX 81 epifluorescence microscope with 40X (UPlanFLN, NA = 1.30) objective lens and Roper Scientific Cascade camera was used for live cell imaging. Microscope and motorized stage (MS-2000, Applied Scientific Instrumentation, Inc) were controlled by Micromanager. Chamlide TC incubator system (Live Cell Instrument) was equipped on the stage of the microscope to maintain a cell culture condition $\left(37^{\circ} \mathrm{C}, 5 \%\right.$ 
$\mathrm{CO}_{2}$ ). Biotinylated coverslips were used to immobilize MNAs on the surfaces. NK cells $\left(0.2 \times 10^{6}\right)$ suspended in $100 \mu$ of IMDM (Cellgro) supplemented with $10 \%$ FBS were added with $1 \mu \mathrm{l}$ Alexa Fluor 647 anti-human IFN- $\gamma$ antibody (BioLegend; clone: 4S.B3). Subsequently, time-lapse imaging was performed with a 15 min interval for $4 \mathrm{~h}$. Acquired images were analyzed using Image $\mathrm{J}$.

Statistical analysis. Statistical significance was tested using a standard t-test. Correlation was calculated using a Pearson correlation analysis. All statistical analyses were performed using Prism (GraphPad Software).

\section{SUPPORTING FIGURES}
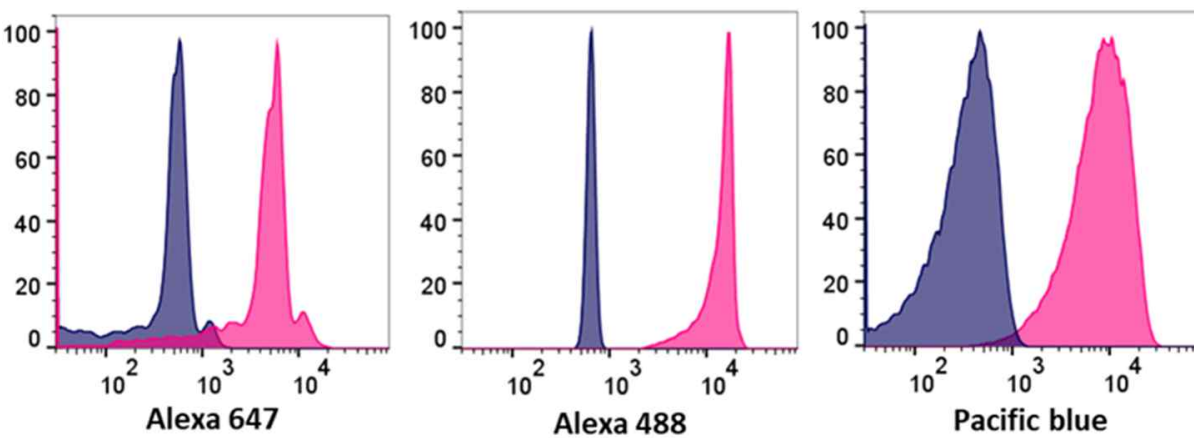

UM (control)

MNA

Supporting Figure S1. MNA functionalization analysis using flow cytometry. Streptavidin labeled with Alexa 647, granzyme B sensor labeled with Alexa 488 were directly analyzed, and all biotinylated antibodies attached on MNAs (anti-NKG2D/2B4/IFN- $\gamma$ ) were mouse IgGs, thus labeled using anti-mouse conjugated with pacific blue. MNAs exhibited substantially higher fluorescence intensities in Alexa 647, Alexa 488, and pacific blue channels compared with unfunctionalized microparticles (UM).

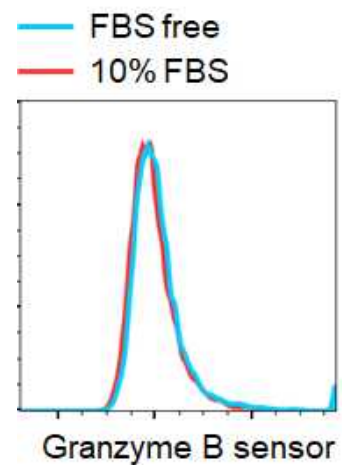

Supporting Figure S2. Effect of FBS on granzyme B sensor fluorescence intensity. MNAs were incubated in full IMDM media containing 10\% FBS or FBS free media for $16 \mathrm{~h}$, and fluorescence intensities were measured by flow cytometry. 


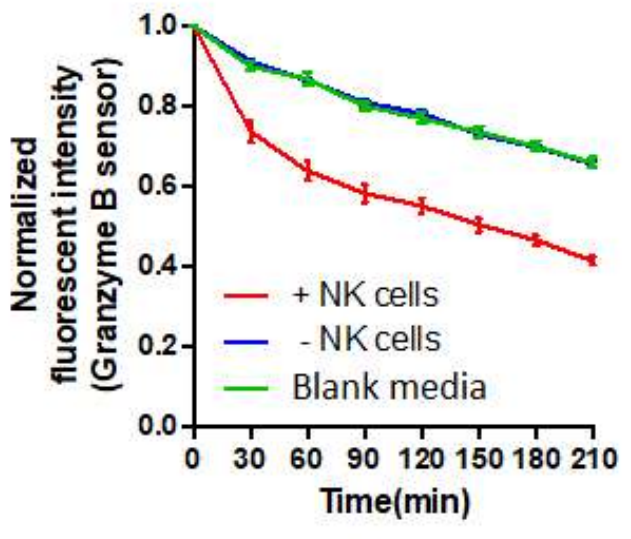

Supporting Figure S3. Effect of photobleaching on granzyme B sensor fluorescence intensity changes in time-lapse imaging. MNAs in IMDM media were added on coverslips, and time-lapse imaging was performed for $4 \mathrm{~h}$ with a 5 min interval to evaluate photobleaching of MNAs by time-lapse imaging. Normalized fluorescence intensities of granzyme B sensor on MNAs were quantitatively analyzed and plotted (green) along with data in Figure 3(B).

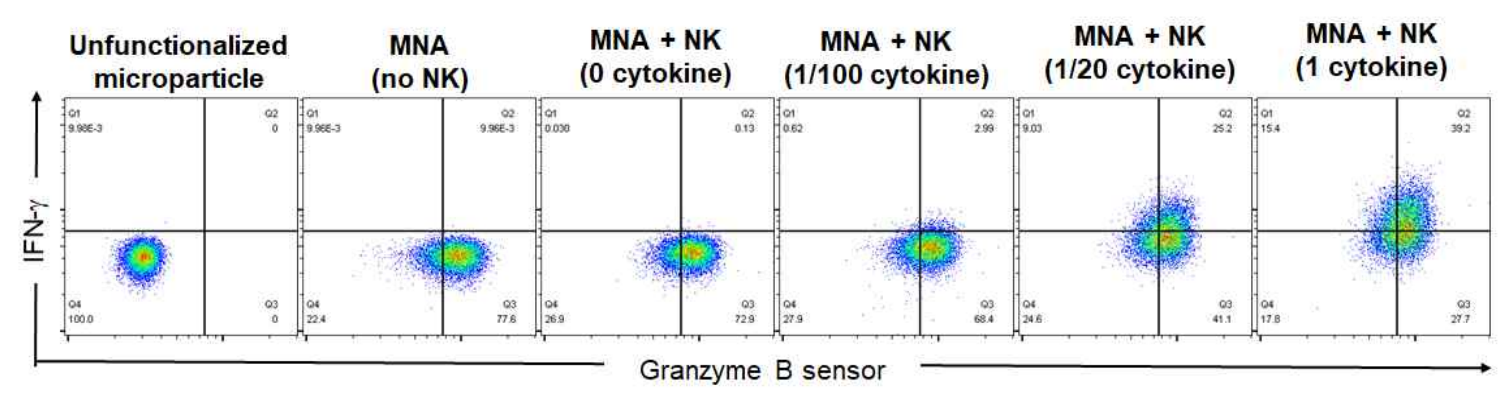

Supporting Figure S4. Representative flow cytometry plots of MNAs mixed with NK cells exhibiting various activities. NK cells with various activities were generated by treating them with various concentrations of cytokine. MNAs were incubated with NK cells for $4 \mathrm{~h}$, and analyzed by flow cytometry. 
- $\left.\left\langle\mathrm{I}_{\mathrm{GBS}}\right\rangle_{0}\right\rangle$ : (geometric) mean granzyme $\mathrm{B}$ sensor (GBS) fluorescence intensity of MNAs

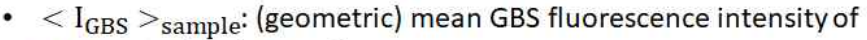
MNA mixed with NK cells

- Fluorescence intensity change of granzyme B sensor on MNA of a given sample $=\left\langle\boldsymbol{I}_{\boldsymbol{G B S}}\right\rangle_{\mathbf{0}}-\left\langle\boldsymbol{I}_{\boldsymbol{G B S}}\right\rangle_{\text {sample }}$

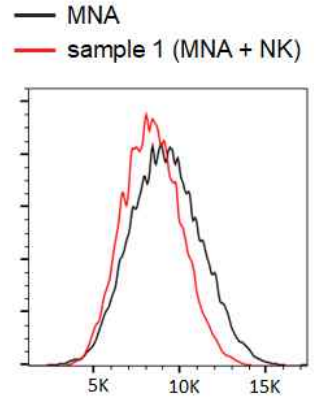

\section{Example}

- $<\mathrm{I}_{\mathrm{GBS}}>_{0}>=9144$

- $<\mathrm{I}_{\mathrm{GBS}}>_{\text {sample1 }}=8342$

- Fluorescence intensity change of granzyme $B$ sensor on MNA of sample1 $=<I_{G B S}>_{0}-<I_{G B S}>_{\text {sample } 1}=802$

Granzyme B sensor (GBS)

Supporting Figure S5. Detailed method for fluorescence intensity change of granzyme B sensor on MNA calculation.

- $<\mathrm{I}_{\mathrm{IFNG}}>_{0}>$ : (geometric) mean IFN- $\gamma$ detection fluorescence intensity of MNAs

- $<\mathrm{I}_{\text {IFNG }}>_{\text {sample: (geometric) mean IFN- } \gamma \text { detection fluorescence }}$ intensity of MNA mixed with NK cells

- IFN- $\gamma$ detection fluorescence intensity change of MNA of a given sample $=\left\langle I_{\text {IFNG }}\right\rangle_{\text {sample }}-\left\langle I_{I F N G}\right\rangle_{0}$

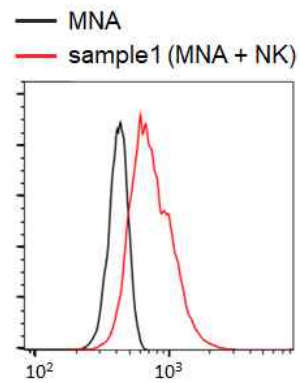

Example

IFN- $\gamma$ detection

- $\left\langle\mathrm{I}_{I F N G}>_{0}>=414\right.$

- $<\mathrm{I}_{I F N G}>_{\text {sample1 }}=755$

- IFN- $\gamma$ detection fluorescence intensity change of MNA of sample1 = $<\boldsymbol{I}_{G B S}>_{\text {sample } 1}-<\boldsymbol{I}_{G B S}>_{0}=341$

Supporting Figure S6. Detailed method for IFN- $\gamma$ detection fluorescence intensity change of MNA calculation. 
0

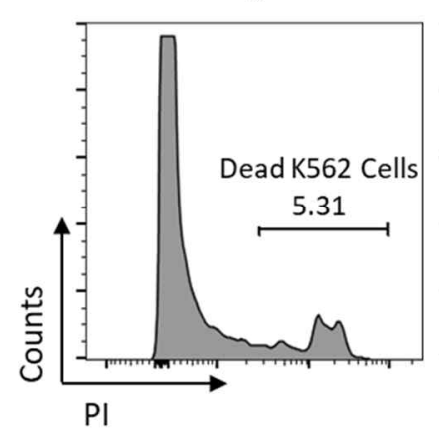

$1 / 100$

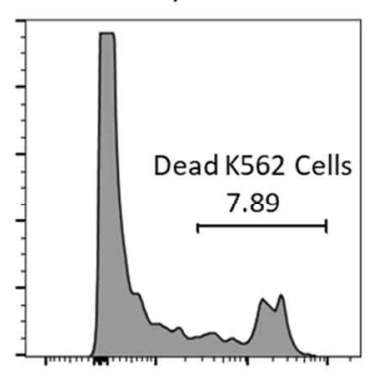

$1 / 20$

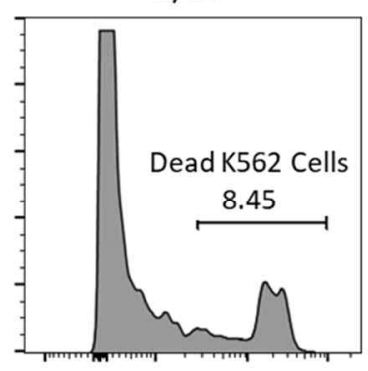

1

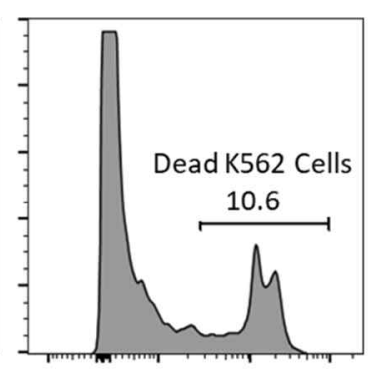

Supporting Figure S7. Representative flow cytometry data of NK cell cytotoxicity assessed using K562 target cells. NK cells treated with various dilution of cytokines were used.

A

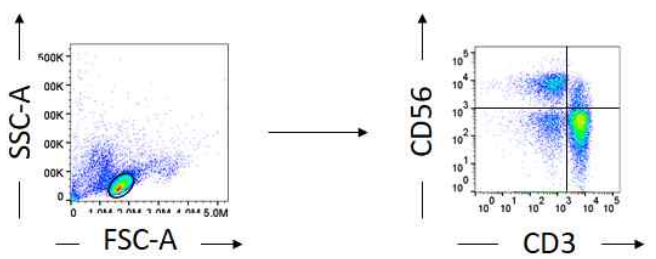

B

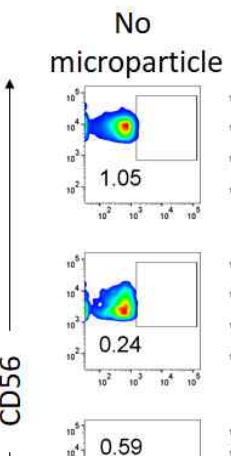

$\mathrm{CD}^{+}{ }^{+} \mathrm{CD} 56$ cells

CD3-CD56- cells
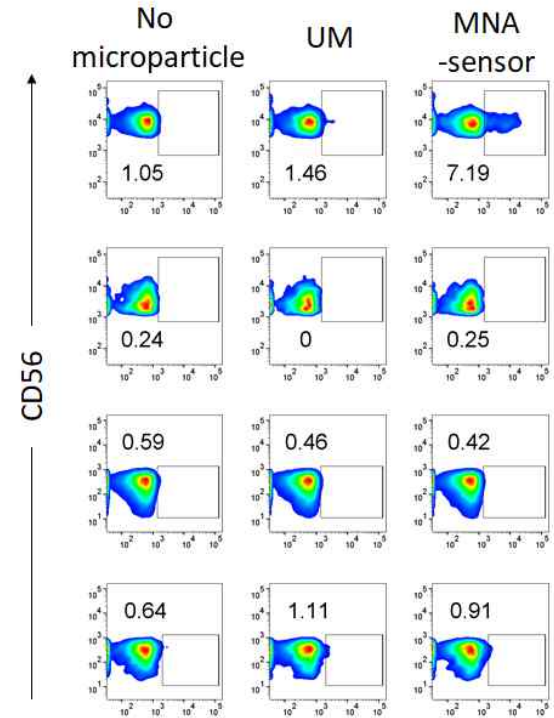

苗

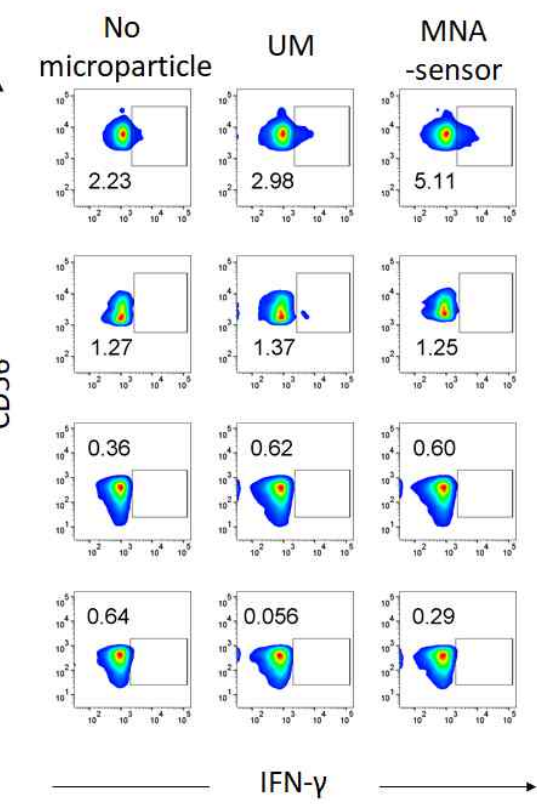

Supporting Figure S8 Selective stimulation of NK cells in peripheral blood monoceuclear cells (PBMCs) using microparticles coated with anti-NKG2D/2B4, or MNAs lacking sensors (MNA-sensor). (A) Representative flow cytometry data showing the strategy for identifying $\mathrm{CD}^{-}{ }^{-} \mathrm{CD} 56^{+} \mathrm{NK}$ cells in PBMCs. (B, C) Representative flow cytometry data showing the percentages of CD107a-positive cells (B) and the percentages of IFN- $\gamma$-positive cells $(\mathrm{C})$ in the indicated cell populations of PBMCs. UM: Unfunctionalized Microparticles. Data are representative of three independent experiments. Cryopreserved PBMCs were thawed, washed twice, resuspended at $2 \times 10^{6}$ cells $/ \mathrm{mL}$ in the presence of $100 \mathrm{U} / \mathrm{mL}$ IL-2, placed in 96- 
well U-bottom plate, and incubated overnight at $37^{\circ} \mathrm{C}$ in a $5 \% \mathrm{CO}_{2}$ incubator. Cytotoxic degranulation was assessed by measuring CD107a expression on the cell surface. Briefly, PBMCs were stimulated with MNA-sensor for $2 \mathrm{~h}$ at $37^{\circ} \mathrm{C}$, and stained with anti-CD3-PerCP, anti-CD56-PE, and anti-CD107a-FITC. After separating MNA-sensors using a magnet, cells were analyzed by flow cytometry. The forward and side scatter (FSC and SSC) characteristics were used to identify lymphocytes, and the CD107a expression on the indicated cell populations within the lymphocyte gate was analyzed using FlowJo. Cytokine production of NK cells was determined by intracellular expression of IFN- $\gamma$. PBMCs were stimulated with MNA-sensor for $1 \mathrm{~h}$ at $37^{\circ} \mathrm{C}$. Thereafter, brefeldin A (GolgiPlug; BD Bioscience) and monensin (GolgiStop; BD Bioscience) were added, and the samples were incubated for an additional $5 \mathrm{~h}$, for a total of $6 \mathrm{~h}$. Then, the cells were first stained with anti-CD3-PerCP and anti-CD56-PE, washed twice, fixed and permeabilized with BD Cytofix/Cytoperm solution (BD Bioscience), washed twice, and stained with anti-IFN- $\gamma$-FITC. Then, the stained cells were analyzed using flow cytometry after separating MNA-sensors using a magnet.

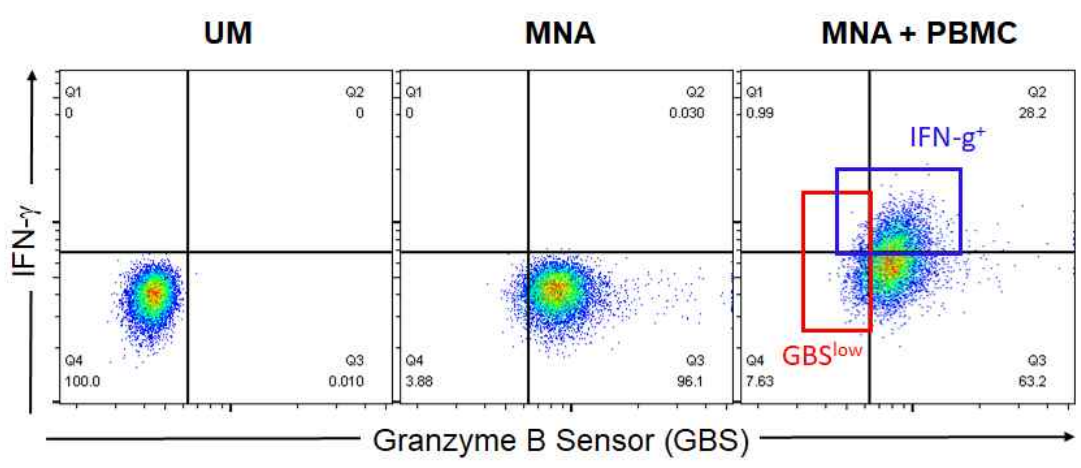

Supporting Figure S9 MNA-based NK cell activity assays using peripheral blood monoceuclear cells (PBMCs). UM: Unfunctionalized Microparticles. Data are representative of three independent experiments. PBMCs in 96-well U-bottom plate were mixed with MNAs in the presence of PE anti-human IFN- $\gamma$ antibody $(2 \mu \mathrm{g} / \mathrm{ml})$ and IL-2 $(100 \mathrm{U} / \mathrm{mL})$. The plate was incubated for $16 \mathrm{~h}$ at $37^{\circ} \mathrm{C}$ in a $5 \% \mathrm{CO}_{2}$ incubator. MNAs were separated using a magnetic stand (DynaMag, Invitrogen), washed twice, and analyzed by flow cytometry.

\section{References}

1. Kweon, S.; Phan, M.-T. T.; Chun, S.; Yu, H.; Kim, J.; Kim, S.; Lee, J.; Ali, A. K.; Lee, S.-H.; Kim, S.-K.; Doh, J.; Cho, D., Expansion of Human NK Cells Using K562 Cells Expressing OX40 Ligand and Short Exposure to IL-21. Frontiers in immunology 2019, $10,879-879$. 


\section{SUPPORTING MOVIE LEGEND}

Supporting Movie S1. A representative movie showing MNA-NK cell interaction. Differential interference contrast (DIC), granzyme B sensor fluorescence (green), detecting IFNantibody fluorescence images are overlayed. 